\title{
A FRAMEWORK FOR THE INTERCONNECTION OF CONTROLLER AREA NETWORK (CAN) BASED CRITICAL CARE UNITS
}

\author{
Nadia Ishaque ${ }^{1}$, Noveel Azhar ${ }^{2}$, Atiya Azmi $^{3}$, Umm-e-laila $^{1}$, Ayesha Urooj $^{2}$ \\ ${ }^{1}$ Department of Computer Engineering, Sir Syed University of Engineering and \\ Technology, Karachi, Pakistan \\ 786. nadya@gmail.com \\ ulaila2002@gmail.com \\ ${ }^{2}$ Department of Electronic Engineering, Sir Syed University of Engineering and \\ Technology, Karachi, Pakistan \\ smartnoveel02@hotmail.com \\ ${ }^{3}$ Department of Computer Engineering, Yanbu University College, Saudi Arabia \\ at.azmi@gmail.com \\ ${ }^{2}$ Department of Computer Science, Sir Syed University of Engineering and Technology, \\ Karachi, Pakistan \\ aurooj161@yahoo.com
}

\begin{abstract}
Patient monitoring helps increasing the mortality by timely notification of exceeding vital signs. By using the vital sign data the critical care staff can make necessary life saving interventions. This requires the underlying network to be very robust so that timely and error free information flow can be guaranteed. Moreover there is a need for a cost effective and robust network technology for continuous and real- time vital signs monitoring in resource constraint settings in developing countries. In this paper we proposed a system of hospitals with interconnected intensive care units. Each intensive care unit employs Controller Area Network (CAN) as underlying technology for networking of bedside units. The data of these bedside units can be communicated with other hospital using higher level protocols such as Ethernet. This allow the hospital staff to share the health information of the patients with the specialized staff in another hospital to provide better cure to the patient and consequently can increase the mortality.
\end{abstract}

\section{KEYWORDS}

Critical care, Vital signs, CAN Protocol, resource-poor Developing countries

\section{INTRODUCTION}

In the critical care units, the patient bedsides are equipped with devices to continuously inform the intensivists and other medical staff about the current vital signs parameters of the patients. By this information, the paramedic staff can make necessary interventions for the prevention and cure of disease. Nevertheless, this critical care is vital for seriously ill patients but it often prove to be costly for resource poor countries [1]. As a consequence, there is always lack of necessary critical care needed for the people who need it. There is a need to focus on the technological solutions that are affordable and sustainable. In addition to the effective critical care services,

DOI : 10.5121/ijait.2012.2401 
International Journal of Advanced Information Technology (IJAIT) Vol. 2, No.4, August 2012

these countries lack specialized staff and other medical equipments for large number of patients during situations of mass disaster. On account of these, every hospital should provide emergency critical care in coordination with the regional hospitals and other medical entities to do this in more effective way [2].

Patient monitoring helps increasing the mortality by timely notification of exceeding vital signs. This requires the underlying network to be very robust so that timely and error free information flow can be guaranteed. In this regard, the CareSint [3] from Siemens and CareVue [4] from Philips provide complete critical care solutions. These vendor specific solutions are expensive for hospital deployment in poor countries. Hence there is a need for a cost effective and robust network technology for continuous and real-time vital signs monitoring in resource constraint settings in developing countries.

Controller Area Network (CAN) is a low cost, light weight broadcast network protocol [5]. It was designed for automobiles but its applications are now expanded to many other industries including hospital [6]. CAN networks are used also in intensive care units including patient beds, in operating rooms, and in other healthcare equipment [7].

On Account of these issues, we propose a system of interconnected ICUs and the hospitals to provide emergency critical care in coordination with each other. This work proposes efficient and robust patient monitoring systems employed with a very low cost and light weight protocol, Controller Area Network (CAN). These CAN based interconnected ICU network is very low cost and hence suitable for deployment in poor countries. Mass deployment of such type of critical care networks is required to maximize the mortality rate.

The remaining of this paper is organized as follows: Section 2 describes our previous work on patient monitoring and some other related work on health monitoring. Section 3 briefly discusses the Controller Area Network Protocol with some description the CAN data packets. The overall system is described in Section 4. In Section 5, the monitoring period and sampling rates of human vital signs are shown. Section 6 discusses the proposed aggregation scheme used to aggregate the vital signs before they are transmitted to the Central Monitoring Station (CMS). Moreover, the algorithm and technique for separation of the aggregated vital signs, received at the CMS, are shown. Section 7 focuses on some discussions and finally section 8 concludes the paper.

\section{BACKGROUND OF RESEARCH}

In our previous work of VCAN [8] we presented the idea of CAN-based vital signs monitoring system. VCAN based system provides provision of monitoring sign vital sign including Electrocardiogram (EKG), heart rate (HR), blood oxidation $\left(\mathrm{SPO}_{2}\right)$, Stolic Blood Pressure (SBP), diastolic blood pressure (DBP), temperature (temp). These vital signs were aggregated and transmitted to the CMS. This method of aggregation was adopted to efficiently utilize the bandwidth by decreasing the bus load. In this work we utilized the VCAN-based system for a network of interconnected ICUs.

In this section we described some of the research related to health monitoring. There is an implementation of aggregation scheme for sending vital sign data over the network in [9]. In this work, the data of four patients traveling in an ambulance, with medical equipment connected via CAN, is aggregated and sent to the hospital via CAN-UMTS gateway. 
A CAN based home health information system is presented in [10], in which the CAN bus is used for interconnecting smart sensors. This system allows the remote monitoring of elderly and sick people.

The interconnection of ICU network is a new application area. In this work we illustrate the system of interconnected CAN based ICUs via CAN-Ethernet gateways and also elaborate the CAN based ICU network where each bedside act as an aggregation unit. This system will ensure the availability of timely information of patients to keep the doctors updated and to provide them early warnings to make life saving interventions.

\section{Controller Area NeTwork (CAN)}

Controller Area Network (CAN) was developed by Bosch to reduce the bulky wiring, complexity and cost related to the interconnection of the electronic devices in automobiles. It has now become standard for industrial communication.

In CAN network, every node is connected to a single CAN bus hence the messages are broadcasted through each node. Each node access the bus with a very efficient bitwise arbitration mechanism which prevents collisions when multiple nodes simultaneously transmit data. This provides good message latency. In addition to this, CAN offers efficient error detection mechanism. These properties make CAN a robust network for communicating short messages. The Bosch specifications [11] contains all the information about the CAN protocol. Here we discussed briefly about the CAN data frame.

CAN protocol defines four different type of frames i.e. data frame, remote frame, error frame, overload frame. Figure 1 shows the data frame of CAN protocol.

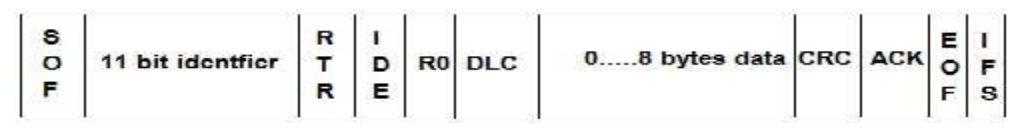

Figure 1. Data frame of CAN protocol

Each data frame can carry 0 to 8 bytes of data. The Data Length Code (DLC) is a 4-bit control field which defines the number of bytes present in the data field of data frame. We used DLC field to identify the type of message which help in applying the algorithm to separate the aggregated data of vital signs.

\section{SYSTEM DESCRIPTION}

In this work we proposed a system in which medical camps are connected with each other and the main hospital to share the health information of the patients. This scenario is best suited in the situation of mass casualties when there is lack of intensivists and other specialized staff. It has been examined that the mortality rate of those patient managed by critical care physician is higher than those managed by non-critical care physician [12]. If the hospitals are networked, the critical care physician in one hospital can remotely give useful directions to the non-critical care staff in distant medical camps. This coordination will able the non-critical care paramedics to provide more effective medication to the patient. Figure 2 shows the scenario of interconnected hospital and camps. 


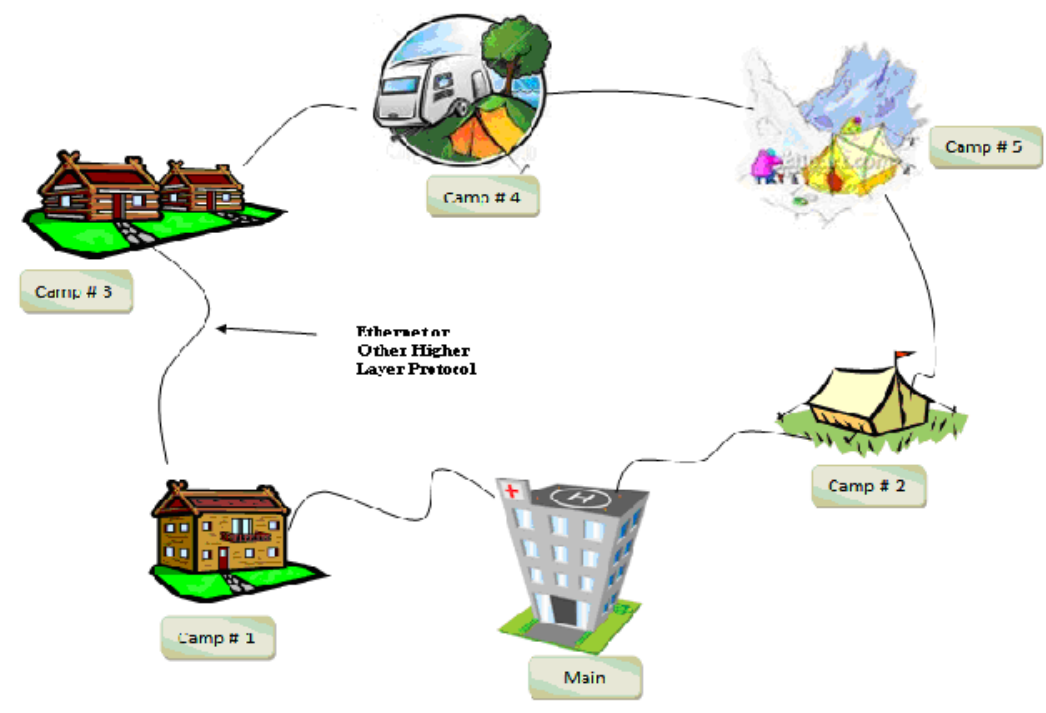

Figure 2. Interconnected Hospital network

The critical care units within short distances can be networked via CAN bus however for transmitting health information data to a distant location, a gateway such as CAN-Ethernet gateway can be used to transmit data of CAN packets over Ethernet.

In order to efficiently utilize the bandwidth we propose to implement the vital sign data aggregation and transmission scheme presented in [13]. According to the VCAN based patient monitoring system, each bedside will act as CAN aggregation unit. The vital signs data from the sensors, attached to each bedside, is encapsulated in CAN data frame by the CAN chip. The identifier of each CAN message will assign an ID to each patient data which help to discriminate the data of patients at the central monitoring station. This monitoring data once received at central monitoring station can be stored and further transmitted to other camps and hospitals.

Figure 3 shows the block diagram of the VCAN based critical care system in which patient bedsides share a single CAN bus. Each node transmits the human vital sign data from the sensors to the Central Monitoring System. Central monitoring system can also put the data on internet so the monitoring of the patient can be done at remote distance via internet.

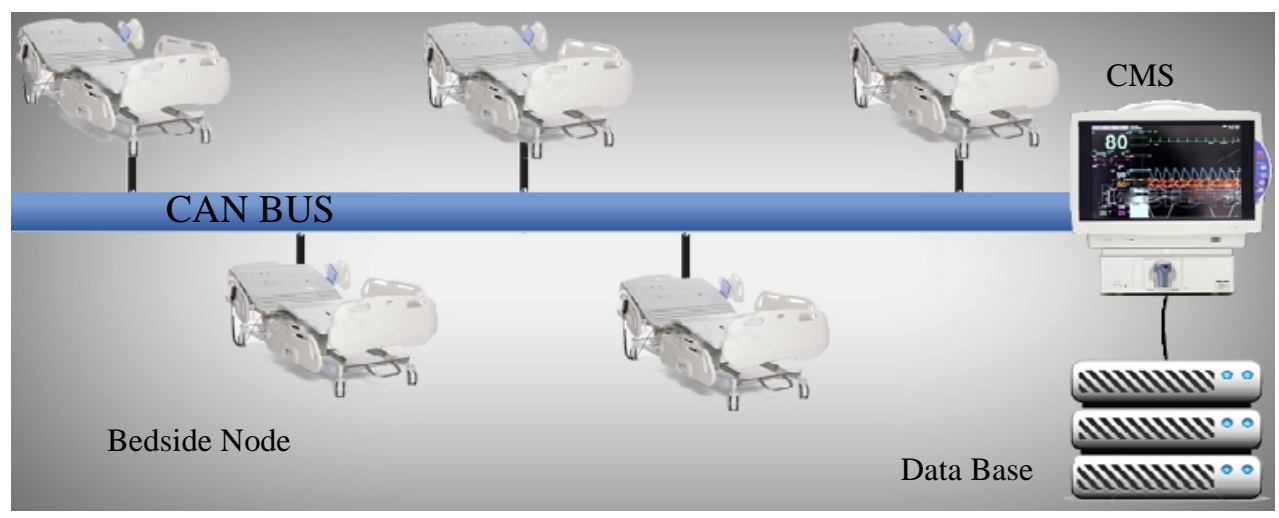

Figure 3: CAN network of Patient Bedside and Central Monitoring System (CMS). 


\subsection{Monitoring Period of Vital Signs}

The proposed system is based on the observation that some vital sign parameters do not change significantly in short period of time such as temperature, stolic and diastolic blood pressure, heart beat and $\mathrm{SPO}_{2}$. Hence, we proposed that data of these sensors be collected periodically after fixed interval of time. However the EKG data will be transmitted continuously at a rate of 300 to 500 samples per minute (typical sampling rates of EKG).

Table 1 shows the monitoring period of each parameter in term of the typical sampling rates.

Table 1. Sampling Rate of Vital Signs

\begin{tabular}{lcc}
\hline Vital Sign & \multicolumn{2}{l}{ Sampling Rate } \\
\hline Electrocardiogram (EKG) & 300 & sample/min \\
\hline Stolic blood pressure (SBP) & 1 & sample/min \\
\hline Distolic blood pressure (DBP) & 1 & sample/min \\
\hline Heart Rate (HR) & 2 & sample/min \\
\hline Temperature (T) & 1 & sample/min \\
\hline Oxygen Saturation (SPO2) & 2 & sample/min \\
\hline
\end{tabular}

\subsection{Bedside Aggregation Unit}

In critical care unit the patient bedside is equipped with sensors to monitor the vital signs. In VCAN each bedside act as an aggregation unit which collects and aggregates the data of sensors in a single CAN packet. Figure 4 shows block diagram of an aggregation units with six multiplexed vital sign sensors.

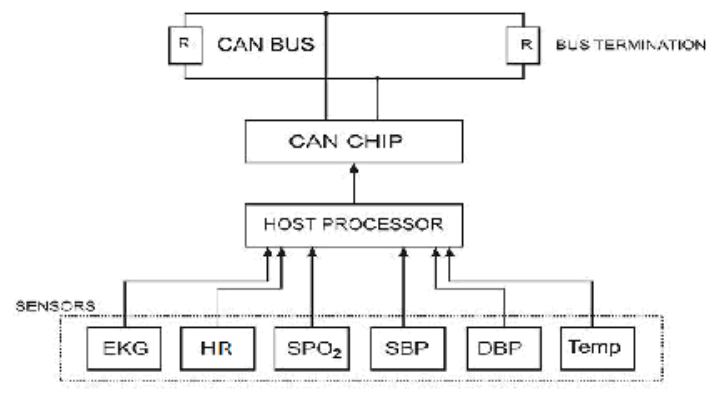

Figure 4. Transmitting CAN Node

The host processor of the CAN node collects digitized sensor data and aggregates it in a single data frame. During the aggregation the node places the data of each sensor in the proposed order according to Table 2 taken from [13]. 
International Journal of Advanced Information Technology (IJAIT) Vol. 2, No.4, August 2012

Table 2. Arrangement of Vital Sign Data in Data Field of CAN Frame

\begin{tabular}{ll}
\hline Byte Number & Vital Sign \\
\hline 1 and 2 & EKG \\
\hline 3 & HR \\
\hline 4 & SPO2 \\
\hline 5 & SBP \\
\hline 6 & DBP \\
\hline 7 & Temp \\
\hline
\end{tabular}

The sampling rate of EKG can vary form 300 to 500 samples per seconds. Figure 5 shows the data transmission of each bedside over a period of 60 seconds.

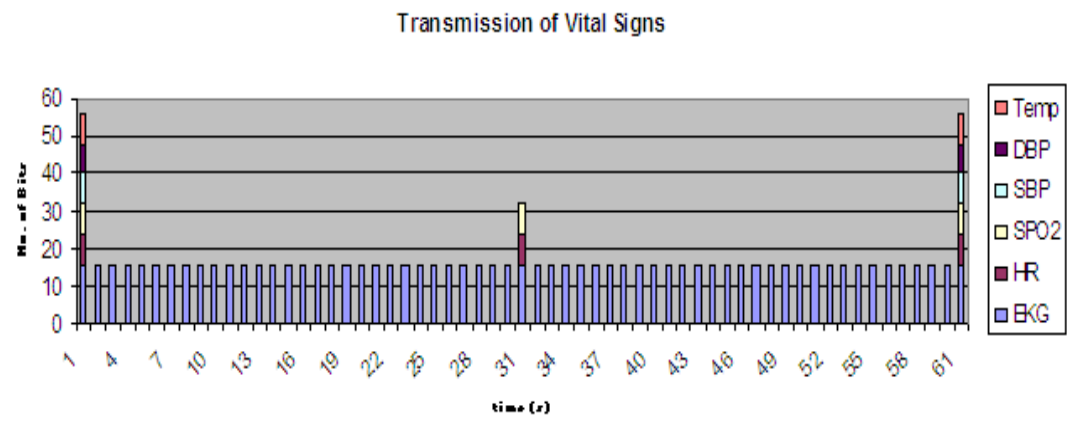

Figure 5. Transmission of Vital Signs

For each message the DLC filed of the CAN protocol provides information about the number of data bytes in the data frame. Since we have restricted the arrangement of the vital sign in data field therefore each CAN data packet can be distinguished by using the information of DLC field. The following section describes the aggregation algorithm for aggregating the vital sign at each bedside.

\subsection{Aggregation and Transmission Algorithm}

Each bedside unit collects and aggregates the sensors data according to the algorithms proposed in [13]. This algorithm uses timers with preset values to schedule the data collection from the sensors. In this way, it avoids frequent transmission of those vital signs which do not change significantly in short interval of time such as temperature, $\mathrm{SPO}_{2}$.

For every patient there is a set of normal range of vital signs parameters which is determined by the doctors. These values are used by the CAN controller for comparison against the measured values of vital signs. If any measured vital sign crosses the prescribed range. The controller sets the "rO" bit of the CAN frame. Figure 6 shows r0 bit in the CAN data frame. 


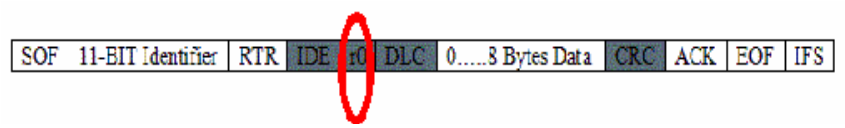

Figure 6. Standard CAN Frame and R0 bit Highlighted

This $\mathrm{r} 0$ bit is reserved by CAN protocol to be used in future. In VCAN this bit is used to indicate the CMS about the abnormal condition of patient. Whenever CMS receives a packet with $\mathrm{r} 0 \mathrm{bit}$ set, it generates the alarm. We have elaborated this process in section 4.4. Flowchart in figure 7 shows the algorithm of vital sign data aggregation and transmission at patient bedside.

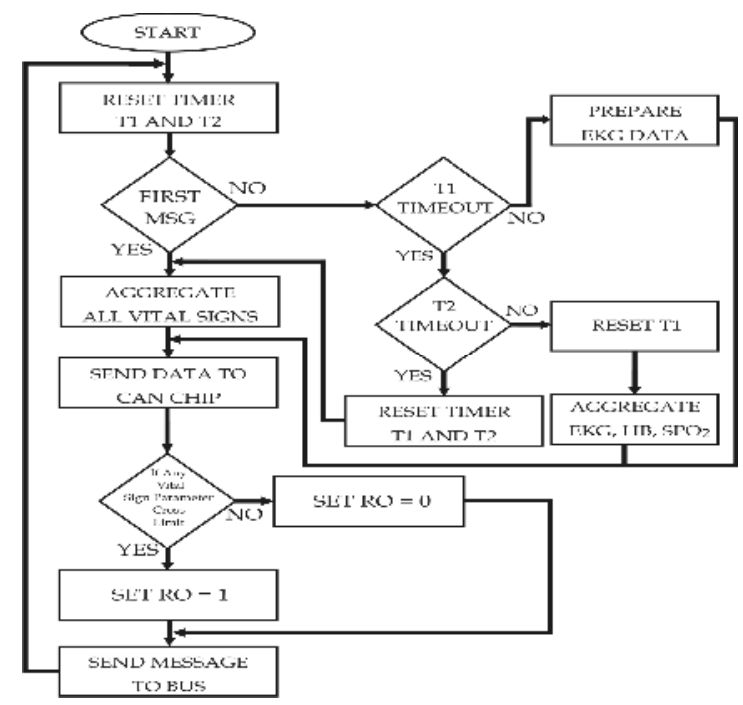

Figure 7. Algorithm for Sending Node at Patient Bedside

\subsection{Data Length Code based Separation of Aggregated Data}

The aggregated data receive at the central monitoring station must be separated in order to extract vital sign data and to process this data to visualize on the monitoring screens. Figure 8 shows the block diagram of data separation unit.

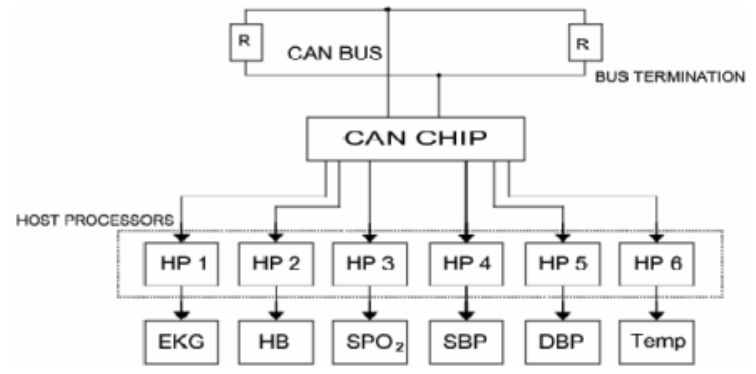

Figure 8. Receiving CAN Node

The CAN controller is responsible for separating the aggregated data received form the CAN bus. Once the data is received, the CAN controller checks the DLC field to extract the information about the length of data. There could only three type of data frames, with value of DLC be 2, 4 or 7 as described in section 3 . 
-- If the value of DLC is 2 then the message contains 2 bytes EKG data only.

-- If DLC is 4, the message contains 2 bytes EKG, 1 byte HR and 1 byte SPO2 data.

-- If DLC is 7, then the data contains data of all vital signs.

In addition to this, the controller check for $\mathrm{r} 0$ bit. If this bit is high, the alarm is generated at the Central Monitoring station to inform the paramedic staff about the anomaly in condition of patient. Fig 9 shows the algorithm of separation of aggregated data at Central Monitoring Station.

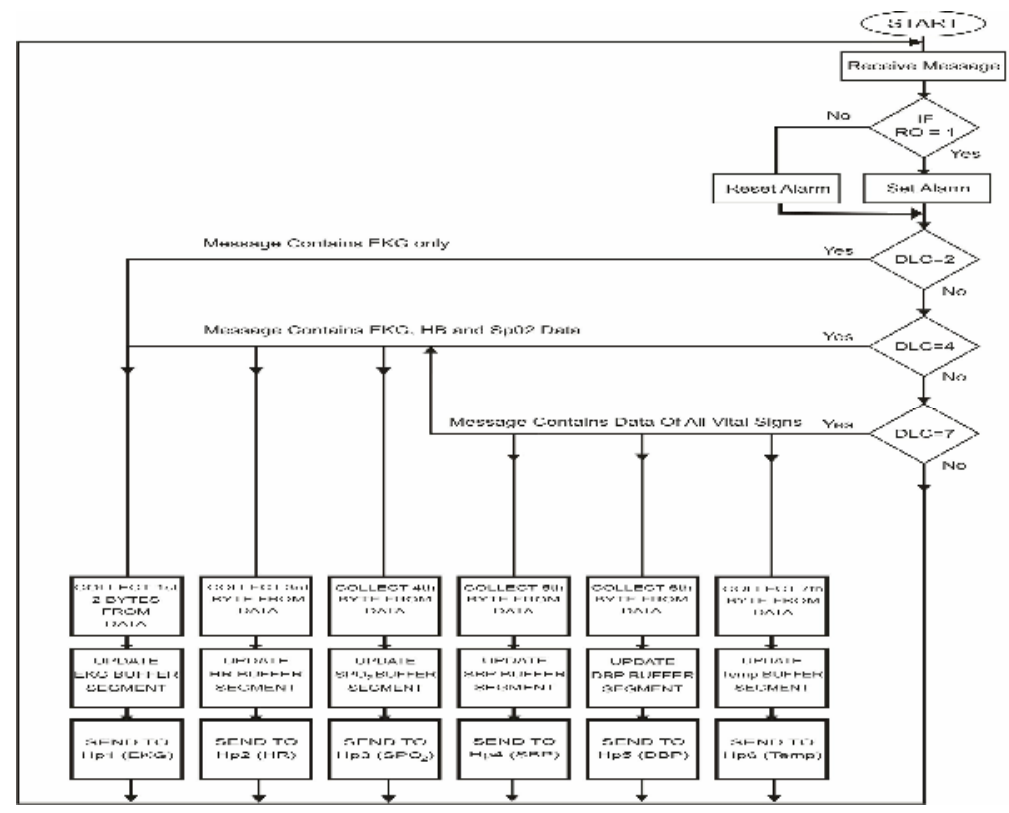

Figure 9. Algorithm for Receiving Node at Central Monitoring Station

\section{ReSults AND Simulations}

In this section we have shown the bandwidth requirement per patient for a VCAN based system. In figure 10 the bar graph shows the bandwidth requirement per patient with and without the aggregation of data. It can be seen that the unaggregated data transmission requires more bandwidth per patient than the aggregated transmission. It can be as high as $50 \mathrm{kbps}$ if EKG is sampled at 500 samples per seconds

Bandwidth Required perpatient

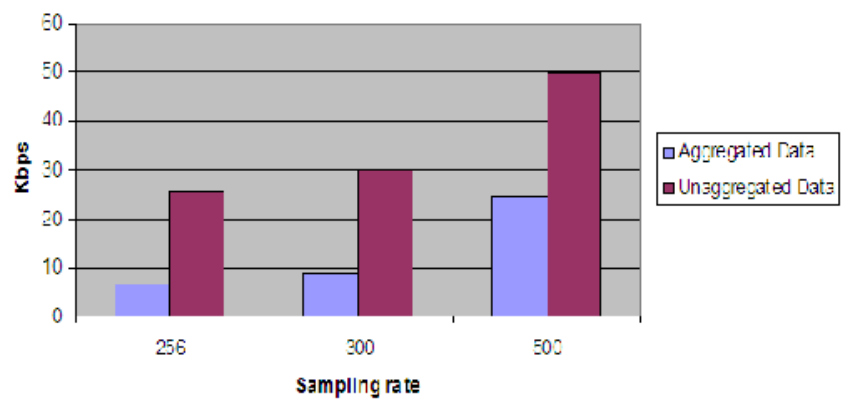

Figure 10. Aggregated Vs Unaggregated Data Transmission over CAN 
CAN provides maximum bandwidth of $1 \mathrm{Mbit} / \mathrm{s}$ when transmission distance is less than 40 meters and can decrease down to $10 \mathrm{kbits} / \mathrm{s}$ for transmission distance of $6 \mathrm{~km}$. In Figure 11, the impact of this bandwidth lost on the number of patient that can accommodated in a single CAN network is shown. For each sampling rate of EKG, the three bar show the number of patients when bandwidth is $1 \mathrm{Mbps}, 800 \mathrm{Kbps}$ and $512 \mathrm{Kbps}$.

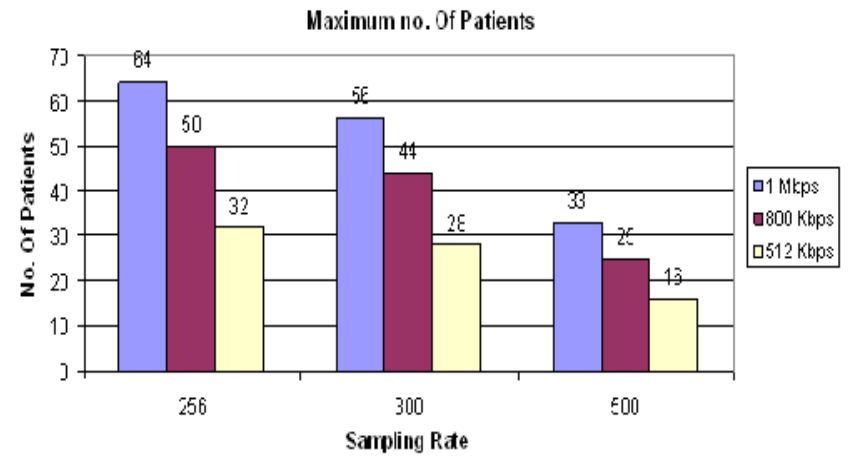

Figure 11. Maximum Number of Patients Accommodated by System with 1Mbps, 800kbps and 512kbps

The proposed system is simulated using Verilogger. It provides visual observation of the output of the transmitting VCAN node. The following figures show the instantaneous results of the simulation. This program generates the values of vital signs and put these vales on the output register. It also compares the generated vital sign value with the normal values and sets or resets the $\mathrm{r} 0$ bit based on the comparison results. The value of DLC indicates the number of bytes sent to the output. Figure 12 and fiure 13 show the simulation results of EKG transmission on Verilog.

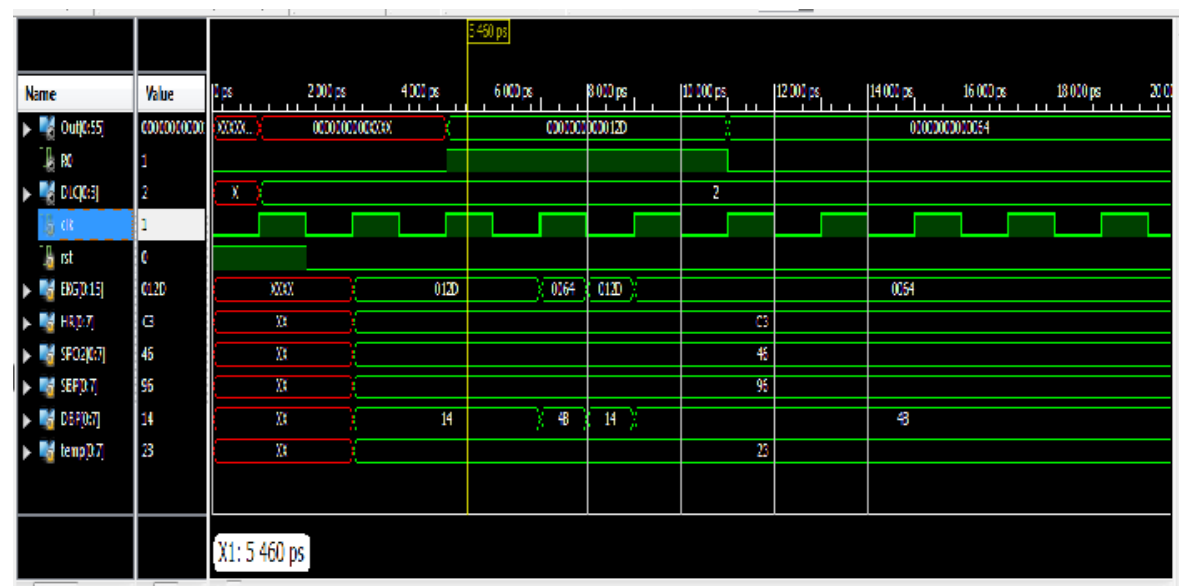

Figure 12. Verilog Simulation Result showing EKG Transmission (DLC = 2) 
International Journal of Advanced Information Technology (IJAIT) Vol. 2, No.4, August 2012

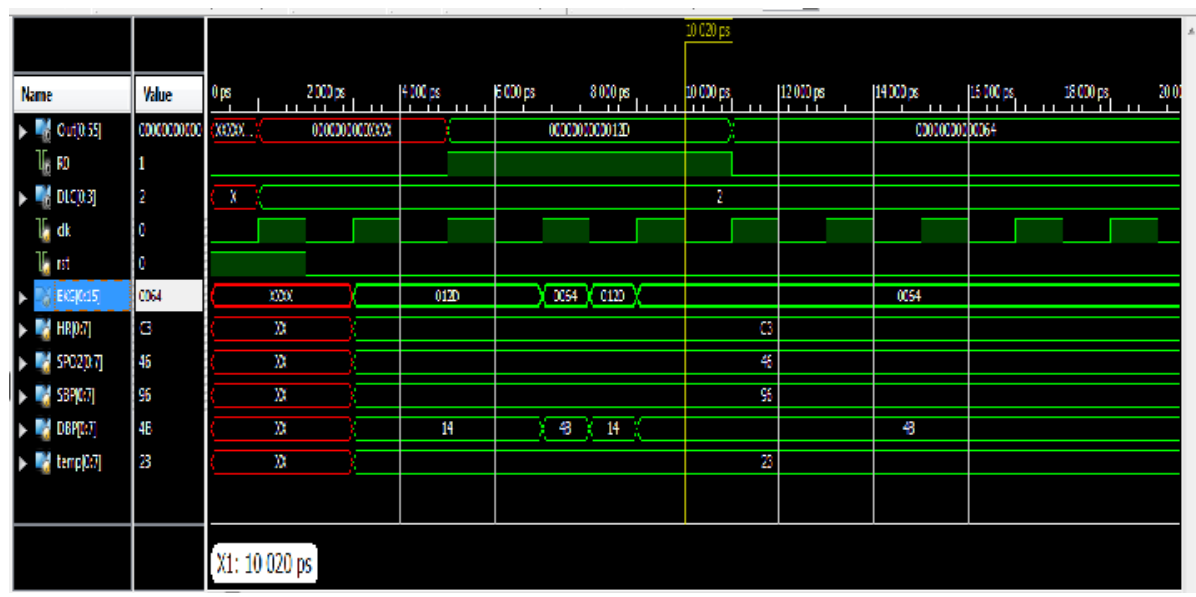

Figure 13: Verilog Simulation Result with r0 = 1

\section{Conclusions}

In this research we proposed a very cost effective solution of patient monitoring in critical care unit. The interconnection of critical care units allows the hospital to share the heath information of the patients so that the critical care physician in one hospital can manage the patients at other hospital so that the mortality can be increased. The deployment of CAN as underlying network in ICU is proved to very low cost and robust. The proposed aggregation scheme has decreased the bus load by $70 \%$ and requires $50 \%$ less bandwidth per patient as compared to unaggregated transmission.

These types are ICUs and critical care network is best suited for resource poor countries. In current era when there is lack of critical care units even in developed countries, the proposed solution will provide a cost effective solution to increase the ratio to intensive care units beds per population.

\section{REFERENCES}

[1] Lewis Rubinson, John L. Hick, Dan G. Hanfling, Asha V. Devereaux, Jeffrey R. Dichter, Michael D. Christian, Daniel Talmor, Justine Medina, J. Randall Curtis and James A. Geiling, (2008) "Definitive Care for critically Ill During a Disaster: A framework for Optimizing Critical Care Surge Capacity”, Official journal of the American College of Chest Physicians, Chest 2008;133;18S-31S

[2] Riviello, Elisabeth D. MD; Letchford, Stephen (2011) "Critical care in resource-poor settings: Lessons learned and future directions", Journal of Society of Critical Care Medicine and Lippincott Williams \& Wilkins, Volume 39, Issue 4, pp 860-867.

[3] Marleen de Mul, Marc Berg, Jan A. Hazelzef (2004) "Clinical information systems: CareSuite from Picis", Journal of Critical Care, Volume 19, Issue 4, pp 208-214.

[4] CareVue-Philips Software System (2007). http://www.medical. philips.com. Accessed june 2012

[5] Hanxing Chen, Jun Tian, (2009) "Research on the Controller Area Network", International Conference on Networking and Digital Society, Volume: 2, pp 251 - 254.

[6] Controller Area Network (CAN) Overview-National Instrument Developer Zone, http://zone.ni.com/devzone/cda/tut/p/id/273. Accessed june 2012

[7] CAN in automation (CiA), http://www.can-cia.org/index.php?id=60, Accessed june 2012

[8] Atiya Azmi, Nadia Ishaque, Ammar Abbas and Safeeullah Soomro, (2011) "Controller Area Network Based Human Vital Sign Data Transmission Protocol”, Advances in Computer Science, Environment, 
International Journal of Advanced Information Technology (IJAIT) Vol. 2, No.4, August 2012

Ecoinformatics, and Education International Conference, CSEE 2011, Volume 214,pp 290-296, Springer

[9] Junaid A. Zubairi (2009) "Aggregation Scheme Implementation for Vital Signs Data over the Network", 6th International Symposium on High-Capacity Optical Networks and Enabling Technologies (HONET), IEEE, pp 129-133.

[10] Gilles Virone, Norbert Nourey, Jean-Pierre, Thomesse, Vincent Rialle and Jacques Demongeot, (2003) "Home Health Information System Based on the CAN Field Bus", 5th IFAC Conference On Fieldbus System and Their Application, pp 23-27

[11] Bosch CAN specification version 2.0 (2011) https://www.esd.cs.ucr.edu/webres/can20.pdf Accessed May 2011

[12] Gregory A Watson. \& Louis H Alarcon (2008) "Intensivists: don’t quit your day job yet” EvidenceBased Medicine Journal Club, edited by Eric B Milbrandt.

[13] Gregory A Watson. \& Louis H Alarcon (2008) "Vital Sign Data Aggregation and Transmission over Controller Area Network (CAN" Evidence-Based Medicine Journal Club, edited by Eric B Milbrandt.

\section{Authors}

Nadia Ishaque, earned Masters in Information Technology at Humdard University, Pakistan. She is Assistant Professor in Computer Engineering Department at Sir Syed University of Engineering and Technology, Karachi, Pakistan. Her research interests include, Controller Area Network Applications and localization in wireless sensor networks. Nadia Ishaque has published three research papers in international conferences and currently working on localization in wireless sensor networks.

Noveel Azhar, has completed Bachelors in Electronic Engineering from Sir Syed University of Engineering and Technology, Pakistan and now working as Research Assistant in Electronic Engineering Department at Sir Syed University.

Umm-e-Laila, has done her bachelors in Science in 2001 and Master in 2007. She is working as assistant professor in Computer Engineering Department at Sir Syed University, Karachi, Pakistan.

Ayesha Urooj, has done Master of Science in Telecommunication \& Networking and conducting research to find the appropriate scheduling algorithm to cope with network and radio conductors and enhance the performance of HSDPA network in terms of spectrum efficiency.

Atiya Azmi, is lecturer in Yanbu Univerisity college, Saudia Arabia. She earned Masters in Telecommunication. Her Research interests are mobile communication, data communications, optical communications and networks.
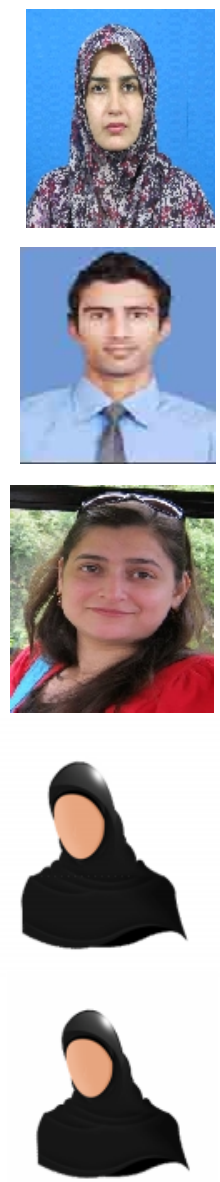